\title{
Controle Semi Ativo de Caos em um Sistema de Amortecedor de Massa Sintonizado Acoplado a um Oscilador Linear e a uma Fonte de Excitação Não Ideal
}

\section{Fábio Roberto Chavarette ${ }^{1}$}

Departamento de Matemática, UNESP - Univ. Estadual Paulista, Ilha Solteira, SP

Mara Lucia Martins Lopes ${ }^{2}$

Departamento de Matemática, UNESP - Univ. Estadual Paulista, Ilha Solteira, SP

\section{Douglas da Costa Ferreira ${ }^{3}$}

Departamento de Engenharia Mecânica, Universidade Federal do Mato Grosso, UFMT, Rondonópolis/Guiratinga, SP

Nelson José Peruzzi ${ }^{4}$

Departamento de Ciências Exatas, UNESP - Univ. Estadual Paulista, Jaboticabal, SP

Resumo. Neste trabalho aborda-se o problema de minimização de vibrações em sistemas mecânicos que utilizam absordores dinâmicos não lineares. Para simular as vibrações excessivas no sistema mecânico e acoplado um motor desbalanceado que causa uma instabilidade caotica no sistema. Uma alternativa para minimizar estas vibrações instaveis é o controle semi-ativo, sendo que neste trabalho nós adotamos para o controle semi-ativo o amortecedor magneto reológico com o objetivo de minimizar as vibrações e reduzir o movimento oscilatório do sistema a um ponto estável. Palavras-chave. Controle Semi Ativo, Caos, Amortecedor Magneto Reológico, Excitação Não Ideal, Vibrações

\section{Introdução}

As estruturas, de modo geral, estão sujeitas a vibrações mecânicas indesejáveis que implicam em um mal funcionamento e até mesmo em sua integridade, causando desconforto a operadores e usuários. Devido a este fato, surge a necessidade de se controlar os níveis de vibração estrutural proporcionando assim segurança e conforto para os operadores e usuários.

O controle estrutural é uma tecnologia para proteção de estruturas que promove uma alteração nas propriedades de rigidez e amortecimento da estrutura pela adição de dispositivos externos ou

\footnotetext{
${ }^{1}$ fabioch@mat.feis.unesp.br

${ }^{2}$ mara@mat.feis.unesp.br

3 dcferreira@ufmt.br

${ }^{4}$ peruzzi@fcav.feis.unesp.br
} 
pela ação de forças externas. Estes dispositivos de controle podem ser classificados como passivos, ativos, híbridos e semi-ativos. $[4,6]$

O controle passivo consiste de aparelhos instalados na estrutura que não utilizam energia proveniente do exterior. São exemplos deste tipo de intervenção o isolamento de base e os amortecedores de massas sintonizadas, também conhecidos como "tuned mass dampers"(TMD).

O objetivo deste trabalho é controlar as vibrações excessivas em uma estrutura causadas por um motor não ideal $[2,7,8]$. A estrutura objeto de estudo é um amortecedor de massa sintonizada [9] acoplado a um motor não ideal [2,8], o qual causa vibrações excessivas ao sistema. Como forma de controlar essa vibrações é proposto o controle semi-ativo através da utilização de amortecedores magneto reológico o qual reduz as vibrações do sistema a um ponto estável [3].

Este trabalho está organizado como a seguir. Na seção 2 apresenta-se o modelo matemático proposto por Frahm [4] com o acoplamento com o motor não ideal. O projeto de controle semiativo, bem como as simulações númericas estão na seção 3 . Na seção 4 apresenta-se as conclusões deste trabalho e logo em seguinta as referencias utilizadas e os agradecimentos.

\section{Modelo Matemático Proposto}

Uma introdução ao problema matemático que se propõe, foi introduzido por Frahm [4] sendo o conceito do amortecedor de massa sintonizado (TMD) considerando um acoplamento linear composto de uma massa e uma mola acoplado a um oscilador linear conservador (OL) (ver Figura 1). Este amortecedor é eficaz numa pequena gama de frequências centrada na frequência natural do oscilador linear conservador.

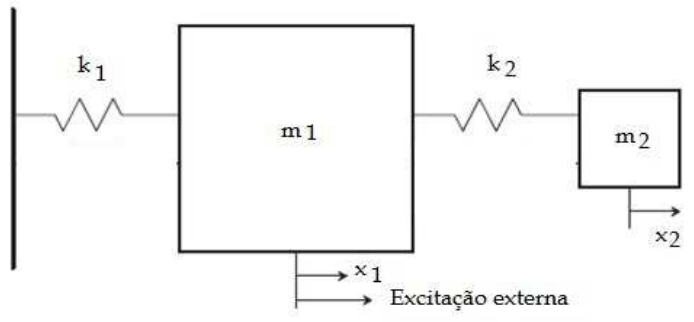

Figura 1: Amortecedor de massa sintonizado acoplado a um oscilador linear [9]

As equações relacionadas com o sistema representado na Fig. 1 e submetidos a uma excitação forçada, são dadas por [9]:

$$
\begin{array}{r}
m_{1} \ddot{x}_{1}+k_{1} x_{1}-k_{2}\left(x_{1}-x_{2}\right)=S, \\
m_{2} \ddot{x}_{2}+k_{2}\left(x_{2}-x_{1}\right)=0
\end{array}
$$

onde $S$ representa a excitação externa no sistema, que neste caso é o torque resultante de um motor de corrente contínua que impõe a excitação não-ideal, de acordo com a posição angular da massa em rotação desequilibrada representada pela equação a seguir. 


$$
\begin{array}{r}
\ddot{z}+b \dot{z}=r \ddot{x}_{1} \sin z+a \\
S=d\left(\dot{z}^{2} \cos z+\ddot{z} \sin z\right)
\end{array}
$$

Acoplando o sistema (1) ao sistema (2) temos:

$$
\begin{array}{r}
m_{1} \ddot{x}_{1}+k_{1} x_{1}-k_{2}\left(x_{1}-x_{2}\right)=d\left(\dot{z}^{2} \cos z+\ddot{z} \sin z\right) \\
m_{2} \ddot{x}_{2}+k_{2}\left(x_{2}-x_{1}\right)=0 \\
\ddot{z}=-b \dot{z}+r \ddot{x}_{1} \sin z+a
\end{array}
$$

Reescrevendo as equações do sistema dinâmico na forma de estado, fazendo $y_{1}=x_{1}, y_{2}=$ $\dot{x_{1}}, y_{3}=x_{2}, y_{4}=\dot{x_{2}}, y_{5}=z$ e $y_{6}=\dot{z}$, as equações do movimento são:

$$
\begin{array}{r}
\dot{y}_{2}=-\frac{-k_{1} y_{1}-k_{2}\left(y_{1}-y_{3}\right)+d y_{6}^{2} \cos \left(y_{5}\right)+d \sin \left(y_{5}\right)\left(a-b y_{6}\right)}{m_{1}-d r\left(\sin ^{2} y_{5}\right)} \\
\dot{y}_{4}=-\frac{y_{2}}{m_{2}}\left(y_{3}-y_{1}\right), \\
\dot{y}_{5}=y_{6}, \\
\dot{y}_{6}=\frac{r \sin \left(y_{5}\right)\left(-k_{1} y_{1}-k_{2}\left(y_{1}-y_{3}\right)\right)+d y_{6}^{2} r \cos \left(y_{5}\right) \sin \left(y_{5}\right)+d r \sin \left(y_{5}\right)^{2}}{m_{1}-d r\left(\sin ^{2}\left(y_{5}\right)\right)} \\
+\frac{\left(m_{1}-d r\left(\sin ^{2}\left(y_{5}\right)\right)\right)\left(a-b y_{6}\right)}{m_{1}-d r\left(\sin ^{2}\left(y_{5}\right)\right)} .
\end{array}
$$

Onde $y_{1}$ e $y_{2}$ são a velocidade e o deslocamento do amortecedor de massa sintonizado e $y_{3}$ e $y_{4}$ são a velocidade e deslocamento do oscilador linear. Os parametros admensionais utilizados nas simulações numericas são $m_{1}=1, m_{2}=0.05, k_{1}=0.5, k_{2}=0.02$ para o amortecedor de massa sintonizado acoplado ao oscilador linear [9] e $a=5, b=1.5, r=0.3, d=0.2$ para a excitação não ideal [2].

\section{Projeto de Controle Semi Ativo}

No campo do controle de vibrações, novos materiais foram desenvolvidos como atuadores e sensores possibilitando o projeto de controladores mais robustos e adaptativos às variações temporais e/ou paramétricas. Destacam-se aqui dois destes, sendo os piezelétricos que atualmente são utilizados como atuadores ou sensores e os fluidos magneto reológicos (MR). Um modelo que é numericamente tratável e vem sendo usado extensivamente para modelar sistemas que contém histerese é o modelo de Bouc-Wen. O modelo de Bouc-Wen é extremamente versátil e pode exibir 
uma grande variedade de comportamento da histerese [1,5]. Neste modelo, a força F do sistema é determinada pela relação a seguir:

$$
F=c_{0} \dot{x}+k_{0} x+\alpha_{0} z_{1}
$$

onde $z_{1}$ obtido pela equação:

$$
\dot{z_{1}}=-\varsigma|\dot{x}| z_{1}\left|z_{1}\right|^{n-1}-\varepsilon \dot{x}\left|z_{1}\right|^{n}+\Lambda \dot{x}
$$

Sendo $\alpha_{0}$ o deslocamento inicial, $c_{0}$ o coeficiente de amortecimento viscoso e $k_{0}$ o coeficiente de rigidez. $\Lambda, \varepsilon, \varsigma$ e $n$ dependem das características do amortecedor.

$$
\begin{array}{r}
F=\frac{3.2}{\left(3 e^{-3.4 i}\right)+1} \dot{x}+k_{0} x+\frac{8.5}{\left(1.28 e^{-3.9 i}\right)+1} z_{1} \\
C(i)=\frac{3.2}{\left(3 e^{-3.4 i}\right)+1} \dot{x}+k_{0} x+\frac{8.5}{\left(1.28 e^{-3.9 i}\right)+1} z_{1}-F
\end{array}
$$

A Figura 2, ilustra o modelo proposto com o amortecedor magneto reologico.

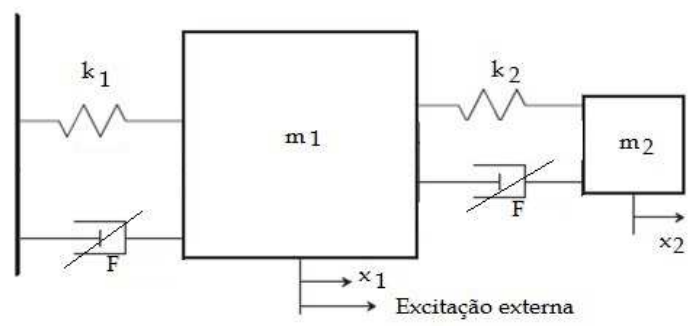

Figura 2: Amortecedor de massa sintonizado acoplado a um oscilador linear excitado por uma fonte não ideal.

Reescrevendo a equação (4) acoplando o amortecedor magneto relógico proposto pela equação (5), a equação do movimento que representa o sistema controlado é: 


$$
\begin{array}{r}
\dot{y}_{1}=y_{2}, \\
\dot{y}_{2}=-\frac{-k_{1} y_{1}-k_{2}\left(y_{1}-y_{3}\right)+d y_{6}^{2} \cos \left(y_{5}\right)+d \sin \left(y_{5}\right)\left(a-b y_{6}\right)}{m_{1}-d r\left(\sin ^{2} y_{5}\right)}-F \\
\dot{y}_{4}=-\frac{y_{2}}{m_{2}-}\left(y_{3}-y_{1}\right)-F, \\
\dot{y}_{5}=y_{6}, \\
\dot{y}_{6}=\frac{r \sin \left(y_{5}\right)\left(-k_{1} y_{1}-k_{2}\left(y_{1}-y_{3}\right)\right)+d y_{6}^{2} r \cos \left(y_{5}\right) \sin \left(y_{5}\right)+d r \sin \left(y_{5}\right)^{2}}{m_{1}-d r\left(\sin ^{2}\left(y_{5}\right)\right)} \\
+\frac{\left(m_{1}-d r\left(\sin ^{2}\left(y_{5}\right)\right)\right)\left(a-b y_{6}\right)}{m_{1}-d r\left(\sin ^{2}\left(y_{5}\right)\right)} .
\end{array}
$$

As características da variação da força de amortecimento, dependendo da velocidade do pistão do amortecedor e aplicada a corrente elétrica na bobina com os parâmetros $\Lambda=180, k_{0}=0, \varepsilon=0$, $n=2$ e $\varsigma=0.1$ dadas em [8]. As trajetorias do sistema com e sem a ação do amortecedor magneto reológico são ilustradas na figura a seguir.
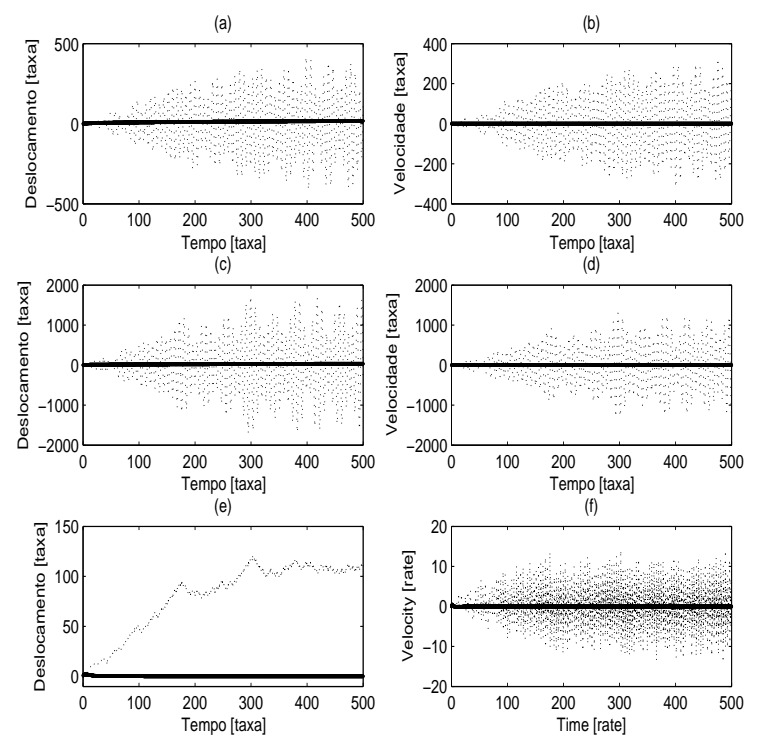

Figura 3: Histórico no Tempo Não Controlado (Linha Pontilhada) e Controlado (Linha Continua). Amortecedor de massa sintonizado. (a)Deslocamento $\left(y_{1}\right)$; (b) Velocidade; Oscilador Linear $\left(y_{2}\right)$ (c) Deslocamento $\left(y_{3}\right)$; (d) Velocidade $\left(y_{4}\right)$; Motor Não Ideal. (e) Deslocamento $\left(y_{5}\right)$ e (f) Velocidade $\left(y_{6}\right)$

As Figuras 3, 4 e 5 mostram o comportamento caótico para o sistema sem controle com as condições iniciais $y_{1}=0.1, y_{2}=0, y_{3}=0, y_{4}=0, y_{5}=1$ e $y_{6}=0$, sendo que para verificar a presença de caos, foi calculado o expoente de lyapunov [10], sendo $\lambda_{1}=0.3978 ; \lambda_{2}=-0.0022$; 
$\lambda_{3}=-0 .-0.030 ; \lambda_{4}=-0.0480 ; \lambda_{5}=-0.0551$ e $\lambda_{6}=-0.055$, caracterizando a presença de caos com um dos seus expoentes positivos.

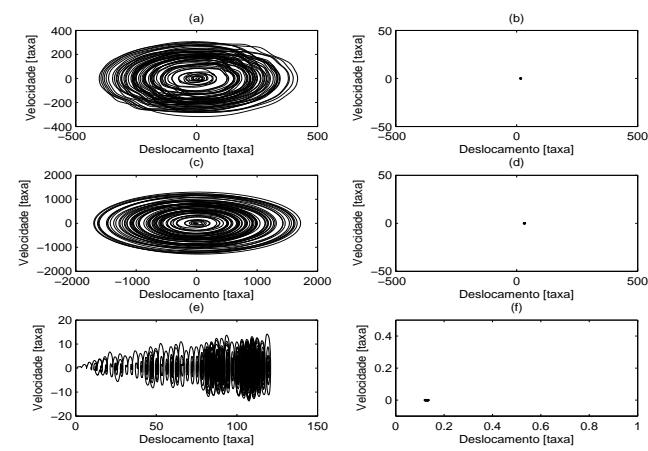

Figura 4: Retrato de Fase.(a) Amortecedor de massa sintonizado não controlado; (b) Controlado; (c) Oscilador Linear Não Controlado; (d) Controlado; (e) Motor Não Ideal Não Controlado e (f) Controlado

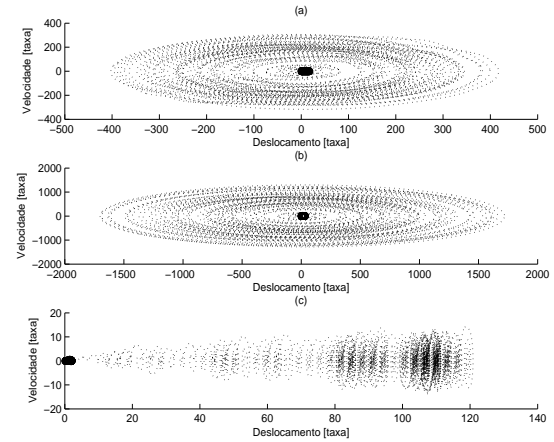

Figura 5: Retrato de Fase. (a) Amortecedor de massa sintonizado não controlado (cinza) e controlado (preto); (b) Oscilador Linear Não Controlado (cinza) e Controlado (preto) e (c) Motor Não Ideal Não Controlado (cinza) e Controlado (preto)

A Figura 5 mostra um compativo entre os retratos de fase contrado e não controlado, demonstrando assim a eficiência do projeto de controle semi ativo aplicado do modelo proposto.

\section{Conclusões}

Neste trabalho foi proposto o acomplamento entre um sistema de amortecedor de massa sintonizado acoplado a um oscilador linear e a uma fonte de excitação não ideal, sendo que a fonte não ideal originou a presença do comportamento caótico no sistema.

Para solucionar as vibrações excessivas derivadas da fonte não ideal, nós propuzemos o acomplamento de um sistema de controle semi ativo. O amortecedor magneto reológico atuou como um eliminador do comportamento caótico conduzindo as oscilações a um ponto estável.

As Figuras 3, 4 e 5 mostram a eficiência deste tipo de controlador para o problema proposto. 


\section{Agradecimentos}

Os autores agradecem o suporte financeiro a Fundação de Amparo a Pesquisa do Estado de São Paulo (FAPESP Proc No. 2014/16807-3) e ao Conselho Nacional de Desenvolvimento Cientifico e Tecnologico (CNPq Proc No. 301769/2012-5).

\section{Referências}

[1] M. O. Bodie and A. Hac, Closed Loop Yaw Control of Vehicles Using Magneto-Rheological Dampers. SAE Technical Paper 2000-01-0107, (2000), DOI:10.4271/2000-01-0107.

[2] F. R. Chavarette, On an optimal linear control of a chaotic non-ideal duffing system. Applied Mechanics and Materials, vol. 138-139, 50-55, (2012), DOI: 10.4028/www.scientific.net/AMM.138-139.50.

[3] B. S. C.Cunha and F. R. Chavarette. Vibration Control Applied in a SemiActive Suspension Using Magneto Rheological Damper and Optimal Linear Control Design. Applied Mechanics and Materials, vol. 464, 229-234, (2013) DOI: http://dx.doi.org/10.4028/www.scientific.net/AMM.464.229.

[4] H. Frahm, A device for damping vibrations of bodies, US Patent 989958, (1911).

[5] A. Hac, I. Youn and H. H. Chen, Control of Suspensions for Vehicles With Flexible Bodies Part II: Semi-active Suspensions. Transactions of the ASME, vol. 118, 518-525, (1996) DOI: 10.1115/1.2801175.

[6] J. P. D. Hartog, Mechanical Vibrations, Dover Books on Engineering, (1985).

[7] A. H. Nayfeh and D. T. Mook, Nonlinear Oscillation. John Wiley and Sons, Inc., (1979).

[8] A. M. Tusset and J. M. Balthazar, On the Chaotic Suppression of Both Ideal and Non-ideal Duffing Based Vibrating Systems, Using a Magnetorheological Damper. Differential Equations and Dynamical Systems, vol. 21, 105-121, (2013), DOI: 10.1007/s12591-012-0128-4.

[9] R. Viquié and G. Kerschen. Nonlinear vibration absorber coupled to a nonlinear primary system: A tuning methodology. Journal of Sound and Vibration, vol. 326, 780-793, (2009), DOI: $10.1016 /$ j.jsv.2009.05.023.

[10] A. Wolf, J. B. Swift, H. L. Swinney and J. A. Vastano, Determining Lyapunov Exponents from a Time Series. Physica D, vol. 16, 285-317 (1985). 